\title{
Nuclear Physics with Magnetic Charges
}

\author{
Robert A. Sizov* \\ Solid State Physics Institute, Moscow Region, Russia \\ Email: sizov.robert@gmail.com
}

How to cite this paper: Sizov, R.A. (2018) Nuclear Physics with Magnetic Charges. Journal of Modern Physics, 9, 145-171. https://doi.org/10.4236/jmp.2018.92010

Received: November 22, 2017

Accepted: January 16, 2018

Published: January 19, 2018

Copyright (C 2018 by author and Scientific Research Publishing Inc. This work is licensed under the Creative Commons Attribution International License (CC BY 4.0).

http://creativecommons.org/licenses/by/4.0/

\begin{abstract}
The magnetic spinor particles (magnetic charges) are the real structural components all varieties of the Mass, for example, atoms, nucleons, positrons and neutrinos. Atomic-shaped device of Mass is the natural and the only possible organization of electric and magnetic charges which can create a gravitational field. At level of a popular language one can define nucleons as "small atoms", and positron and neutrino as "very small atoms". The electric and magnetic fundamental particles in neutron and proton shells which by tradition should be called quarks have charges of smaller magnitude than the charges of particles in atomic shells. Positron which participates in the gravitational interaction and, consequently, has an atomic-shaped device is the most likely candidate for the role of the proton nucleus. The most likely candidate particles on the participation in nuclei of proton and neutron as well as in nuclei of the positron and neutrino are presented in the article. So-called abnormal magnetic moment of neutron is formed by the quark magnetic dipoles which are like to unpaired electrons in the so-called magnetic atoms rotate on the outer orbitals of the neutron shell. The participation of the "magnetic electron" (magneton) in the neutrino core assumes the existence of the so-called anomalous magnetic moment and in the neutrino shell. The existence of real magnetic charges in the structures of the Mass draws our attention on such important problem as interaction between charges in the framework of electromagnetic dipoles such as $\mathrm{e}^{+} \div \mathrm{g}^{-}$and $\mathrm{e}^{-} \div \mathrm{g}^{+}$in which manifest the weak attraction. Weak interaction by its nature is electromagnetic. So-called electromagnetic interaction, manifested in pairs of homogeneous charges of opposite signs, is either electric or magnetic, but not electromagnetic. The explanation of the weak interaction in the marked pairs of charges is based on the author's concept of the World Physical Triad and "Dark Energy". Forces responsible for the interaction of the charges composing the electromagnetic dipoles correspond, conditionally of the weak charges of the particles which what assume mutual suppression of the influence of their fields on the Energo-medium and the formation of the weak "Dark energy".
\end{abstract}

${ }^{*}$ At present works as individual researcher; Dr. of physical and mathematical sciences. 
Complex of magnetic particles, the quark magnetic dipoles and magneton by means of which the interconversion of a proton and a neutron is realized and maintained their constant number in the atomic nuclei can be called as magnetic meson. Namely, a processes of interconversion between a neutron and a proton which, as a rule, are not accompanied by secretions, created the illusion of neutron stability in atomic nuclei. The energy created by an exchange of magnetic mesons between neutron and proton can be a component of nuclear forces (strong interaction). Another effective and, most likely, the main component in the composition of the nuclear forces is the gravitational "Dark Energy". Physics and structure of neutrinos presented in the paper suggest that the nature of these particles closer to the ideology of E. Majorana than P. Dirac's.

\section{Keywords}

Magnetic and Electric Charges, Antielectrons, Magnetons, Antimagnetons, Bispinor, Physical Mass, Vortex Electromagnetic (Gravitational) Field, Quarks, Protons, Neutrons, Positrons, Neutrino, Mesons, Weak and Strong Interactions, "Dark Energy"

\section{Introduction in Physics of Electric and Magnetic Spinor Particles That Make up Atomic Shells}

The magnetic spinor particles (magnetic charges) which are direct sources all of magnetic fields in Nature have been experimentally observed by the author in the structures of atoms and substance and for first time are presented of scientific community in 2001 in the publication [1]. Experiments which prompted the author devote oneself the detection and investigation of real magnetic charges were his neutron diffraction studies of magnetic structures of hexagonal ferrites $\mathrm{Ba}_{2-\mathrm{x}} \mathrm{Sr}_{\mathrm{x}} \mathrm{Zn}_{2} \mathrm{Fe}_{12} \mathrm{O}_{22}$ system which were conducted on atomic reactor ITEP in Moscow (1969-1971) [2] [3]. Significant displacements of the density of so-called magnetic moments of $\mathrm{Fe}^{3+}$ ions from their nuclei (up to $0.9 \AA$ ) discovered in these experiments served as a starting point for assumption of the author about the possible participation of real magnetic charges in structures of atoms and substance. The author managed to carry out a number of experiments, in subsequent years (1972-1992) which confirmed his preliminary assumption. The results and description of the author's main experiments on the problem: real magnetic charges in atoms and substance are given in [1] [2] [3] [4] [5].

Magnetic spinors in compositions of atomic shells (its author's name is magnetons) are fundamental particles of Matter, which by their physical parameters are the magnetic analogs of electrons, i.e. they have magnitudes of charges and spins equal to corresponding values for electrons. These particles in the text of the article can, sometimes, being called as "magnetic electrons". Magnetons, like electrons, have a charge with a negative sign $\left(\mathrm{g}^{-}\right)$they belong to the class of leptons and along their statistical properties to fermions (spin equal 1/2). 
Magnetic antispinors, for example, antimagnetons are true antiparticles to magnetons. They have a positive charge $\left(\mathrm{g}^{+}\right)$and belong to class of antileptons. Antimagnetons are of the antifermions with the antispin $(-1 / 2)$ on relation to spinors.

The basic state of spinor particles is the state of bispinor, i.e. of connected pair of spinor particles with charges opposite signs. Particles in this state are tightly pressed together, what is accompanied by compensation of activity their spins. Such a spinor pair can also be called as enclosed or spin-enclosed dipole.

The above ground state of spinor particles in the form of magnetic or electric bispinors does not lead to particle annihilation. The particle annihilation version was initiated by the results of the electron-positron interaction. However, the positron not a true antielectron, but is a varieties of Mass, i.e. it have atomicshaped structure. The real antielectron "sits" in the positron structure as its nucleus and determines the electric charge of the positron. Upon contact with a positron the electron combines with the true antielectron with forming an electric bispinor, and everything else from the composition of the positron in the form of the shallow electric and magnetic particles, most likely quarks, is ejected away. Gamma quanta, observed in this case, are evidence of the annihilation but, exclusively, of the electromagnetic shell of positron. It is important to note that pairs of charges in state of bispinor are not manifested yourself by spinor fields and are very difficult to detect. When a positron collapses in the so-called annihilation of particles, it is relatively easy to detect only gamma quanta, that and is observed in practice.

Under external influences on bispinors, for example, by means of spinor fields, their spin-enclosed state can be violated with the formation of spinor dipoles. Namely the electric and magnetic bispinors in the form of the corresponding spinor dipoles form of atomic shells and, as will be shown in this paper, the shells of nucleons, positron and neutrino.

Magnetic charges are the real structural components of all varieties of the Physical mass, i.e. of atoms, nucleons, positrons and neutrino. Without the participation of magnetic charges, for example, neither atoms nor substance can be form.

The author has determined three reasons that for almost 200 years (since the discovery of Oersted in 1820) inhibit the recognition of real sources of magnetic field, i.e. magnetic spinor particles (magnetic charges).

1) The main reason is in the confinement physics of magnetic particles in the substance which is radically different from the confinement of electrons. Namely confinement of the magnetic charges in the substance creates serious problems with their detection and identification [5] [6]. It is important to note that in the special conditions of confinement in the substance there are and such real particles as true antielectrons, which have been replaced by such of the theoretical "prosthetics" as the Dirac "holes" or the electron vacancies [7]. The true antielectrons together with electrons form electrical bispinors. As will be shown below these bispinors in the form of electric dipoles in conjunction with magnetic 
dipoles in the shells of atoms participate in the formation of atomic gravitational fields. The used currently electron shell model of atoms, in the light of the existence in her composition, except electrons, of the real magnetic charges and true antielectrons, must give way to the electromagnetic shell.

2) The second reason is Maxwell's erroneous electromagnetic concept, which can be defined how the vicious electric magnetism of Maxwell [8]. This concept is the result of the superficial view of the Great Physicist on the famous Oersted Experience when only obvious factors were taken into account: the electric current and the magnetic field around the conductor. As for the true sources of the magnetic field, that is, real magnetic charges, they turned out to be unnecessary, and then, almost 140 years, the physical theory, more or less successfully, explained all the magnetic and electromagnetic manifestations using exclusively electricity.

3) As the third reason the author considers the subjective factor which also can be to define as the inertia of thinking. As the physical theory plunged into the "swamp" of purely electrical interpretations of numerous magnetic and electromagnetic effects and manifestations it became increasingly difficult for it to perceive of real magnetic charges, since and without them all, more or less, was explained. Under such conditions, the introduction of magnetic charges into basic physical concepts has considered as "harmful action", since it presuppose the elimination of a huge number of theoretical "the air castles" that were built without the participation of real magnetic poles and true antielectrons.

The purpose of this article is the refinement of a structural device of the nucleons, positrons and neutrino, in connection with the new possibilities that are opened by the introduction into physical representations of real magnetic poles (magnetic charges), as well as of true antielectrons. The author's conclusions of the device of these particles allow in new light to consider a number of serious problems related to atomic and nuclear physics.

\section{The Electromagnetic Shells of Atoms are Direct Sources of Gravitational Field}

Based on the results of own experiments as well as the works of other researchers [9], the author came to a very unusual, from the standpoint of modern physical concepts the conclusion, that the atomic shells consist from electric and magnetic charges and are electromagnetic, rather than electronic as it has always been considered. Under this a number of magnetic spinor particles in atomic shells, is approximately, equal to the number of electric particles. Namely is the electromagnetic shells of the atoms are the natural sources (generators) of the gravitational field (GF) which is, in reality, of the vortex electromagnetic field. The elementary source of the gravitational field is the spinor electromagnetic quasi-particle which received the name S-Graviton ( $\mathrm{S}=$ source). Composition of the S-Graviton: two spinors (electron and magneton) and two antispinor corresponding to them. Figuratively speaking the S-Graviton is a combination of 
the electric and magnetic dipols, rotating in antiphase at the same atomic orbital. This quasi-particle can also associate with circular currents of electric and magnetic charges, which move in antiphase on the one orbital.

The model representation of the orbital electromagnetic current or S-Graviton can be written in following form: $\operatorname{rot}\left[\boldsymbol{J}_{e}-\boldsymbol{J}_{g}\right]$, where $\boldsymbol{J}_{e}$ and $\boldsymbol{J}_{g}$ are vectors of the instantaneous density of currents electric (e) and magnetic $(g)$ charges corresponding to their of vortex flows. These currents can also be defined as the vortex (circular) motion of el ectric and magnetic charges in the composition of the corresponding dipoles on the orbitals of atomic shells. Then the equation process gravitational field formation by means the S-Graviton can be presented in the form:

$$
k \operatorname{rot}\left[\boldsymbol{J}_{e}-\boldsymbol{J}_{g}\right]=\operatorname{rot}[\boldsymbol{E}-\boldsymbol{H}]
$$

where $\boldsymbol{E}$ and $\boldsymbol{H}$ are the vectors of the instantaneous electric and magnetic field strengths in the structure of the vortex electromagnetic (gravitational) field, $k$ is the proportionality coefficient. The minus signs indicated in the above equation for the formation of the gravitational field correspond to both the antiphase of the orbital currents of electric and magnetic charges, and the anti-directionality of the vectors of the instantaneous electric and magnetic field strength at each point of the gravitational field.

Note 1. The above-mentioned mutual orientations of the vectors of the electric and magnetic currents in the compositions S-Gravitons and corresponding it orientations of vectors $\boldsymbol{E}$ and $\boldsymbol{H}$ of electric and magnetic fields in the structure of the gravitational field, meet the fundamental principle of minimizing the "Dark energy" or, what is same, corresponds to the principle of least action. Namely minimization of "Dark energy" is a basic physical principle which is responsible for the formation of all types of Physical mass. Read more about the principle of minimizing the "Dark energy" see in [10] [11].

The vector-vortex analogy between magnetic (a) and gravitational (b) field is show on Figure 1. Unlike from the vortex magnetic field to each point of which there corresponds one vector of instantaneous strength of $\boldsymbol{H}$, each point of the elementary gravitational field corresponds to two vectors of instantaneous strength of the fields of $\boldsymbol{E}$ and $\boldsymbol{H}$ equal by value and oriented antiparallel each other. In addition, on Figure 1, it is shown that the vortex magnetic field which determined by the vortex vector $\operatorname{rot} \boldsymbol{H}$ formed the revolved magnetic dipole. The source of the elementary gravitational field is the S-Graviton, i.e. two connected dipoles (electric and magnetic) rotating in antiphase on one atomic orbital. The classical equations of the processes formation of vortex fields: magnetic (a) and electromagnetic (gravitational) field (b) are also down on Figure 1.

If polarization of vortex vectors $\operatorname{rot}\left[\boldsymbol{J}_{e}-\boldsymbol{J}_{g}\right]$ of S-gravitons is realized in the structures of Physical masses (in atoms, nucleons, substance etc.) that is accompanied by polarization of vortex vectors $\operatorname{rot}[\boldsymbol{E}-\boldsymbol{H}]$, then by analogy with magnetic fields of the ferromagnetics, the gravitational fields being emitted by these masses can be called the ferrogravitational fields (FGF). The gravitational 
(a)

(b)

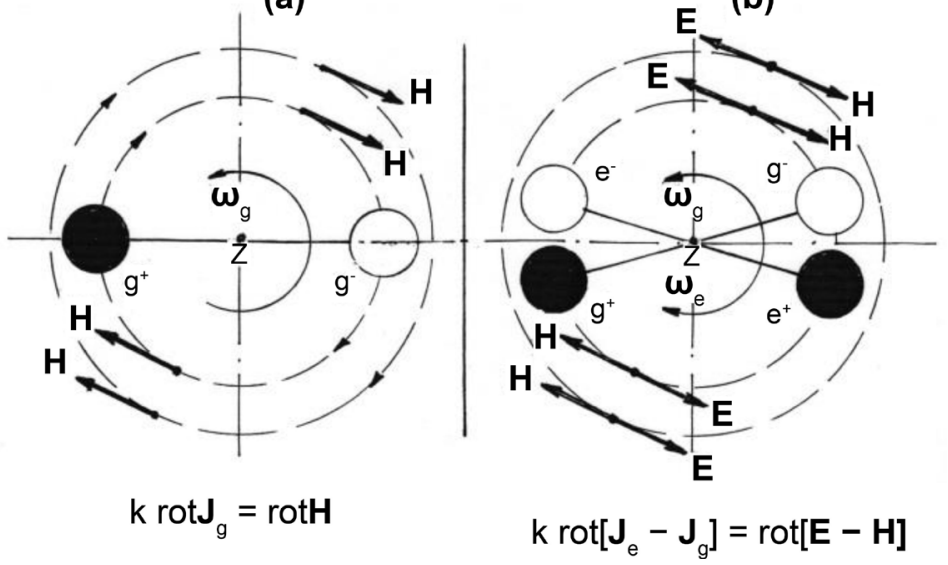

Figure 1. Schemes of the processes formation of vortex spinor fields: of the magnetic field $\operatorname{rot} \boldsymbol{H}$, by means rotating magnetic dipole (a) and gravitational field $\operatorname{rot}[\boldsymbol{E}-\boldsymbol{H}]$, by means S-Graviton (b). By mean of white circles on Figure 1 showed of negative charged electric and magnetic spinors: electrons $\left(\mathrm{e}^{-}\right)$and magnetons $\left(\mathrm{g}^{-}\right)$, and black circles indicate positively charged antispinors corresponding to them with charges $\mathrm{e}^{+}$and $\mathrm{g}^{+}$.

fields formed by physical masses in the absence of polarization of the vortex vectors of S-gravitons in their structures are a tensor or quasi-scalar fields. And again, by outward analogy with magnetism such fields can to define as paragravitational fields (PGF).

The mathematical expression corresponding to the states of the ferrogravitational is have the form $\langle\operatorname{rot}[\boldsymbol{E}-\boldsymbol{H}]\rangle \neq 0$. The field corresponding to the condition: $\langle\operatorname{rot}[\boldsymbol{E}-\boldsymbol{H}]\rangle=0$, by analogy with the paramagnetism can be defined as paragravitational field.

Between the masses (bodies) that emit paragravitational field is implemented a well-known Attraction or Gravity. Since the overwhelming of number physical masses in the Universe emit the paragravitational field, the attraction between them is constituted the famous law of universal gravitation. The physical masses which emit ferrogravitational fields will push off from masses-sources a paragravitational fields, for example, from Earth. This last effect, discovered by the author of the article, called as the effect Gravitational levitation [12] [13] [14] [15].

\section{The Mass Is the Atomic-Shaped Electromagnetic Structure Capable of Emitting a Gravitational Field}

The author in his publications, since 2001, tried to explain that all varieties of Mass are the electromagnetic structures of atomic-shaped type consisting of electric and magnetic spinor particles [1] [5] [16]. Main characteristic properties of all varieties of Mass is ability to emit a gravitational field that is formed as the result joint orbital currents of electric and magnetic charges and is the vortex electromagnetic field.

Thus Mass can called as the electromagnetic spinor structure which is capable of forming the gravitational field. It is important to emphasize that Physical Mass and, for example, Matter are completely different physical categories. The 
Physical masses are, for example, nucleons and atoms. It is especially important to note that the individual spinor particles as electric so and magnetic are massless, because the mass is a result of their joint "structural operations". For example, the electron is massless particle and no "divine bosons" can't give him mass. As noted above, the masses (bodies) emitting the paragravitational field, manifest the so-called gravitational attraction or gravitation to each other, i.e. participate in the gravitational interaction. Since the nucleons, positrons and neutrino are the varieties of the Mass and, as is known attracted to the Earth, there are reasons to assume that they emit the paragravitational field. This very important assumption allowed the author to clarify the structural features of the nucleons and other, as well as identify the main parameters of particles in their compositions.

Note 2. The expression "so-called" to terms: "the attraction of masses", "magnetic moment" or "annihilation of pair" sometimes use in the article in connection with the fact that in reality, masses emitting a paragravitational field not attracted to each other, but are pressed by forces of "Dark Energy". The notion of "magnetic moment", according to the author, is a theoretical prosthesis that was introduced alternative to the existence of the real magnetic poles (magnetic charges) in atomic shells. Questions relating to "annihilation of pair" which very important for disclosing the essence of the problems raised by the author will be repeatedly considered in the article. We note that the essence of the noted a concepts ("attraction" or compression of masses, as well as the "magnetic moment") represent is separate "histories" and, in principle, do not affect the logic of reasoning and the conclusions presented in this article. Therefore we further, in main, will be to use the generally accepted в present time notions: of the "attraction" the masses (bodies) and the "magnetic moments".

\section{The World Physical Triad, Particles and Fields of Matter and Antimatter, "Dark Energy"}

The results of years of research of the real magnetic spinor particles allowed the author to formulate the conception of the World Physical Triad (PT) according to which the real World consists of three fundamental phases: of the Matter, Antimatter and Energo-phase (Energo-medium). Particles Matter are spinors and has charge with a negative sign, and the Antimatter particles are antispinors with positive charge. Particles Antimatter constitute approximately half all of real spinor particles, i.e. charged particles in World, and their absence in the physical representations is determined by Physics their of confinement in the substance. The process so-called annihilation of pair: spinor and antispinor, is accompanied by a strong compression (pressing) of these particles to one another by forces "Dark Energy" and, in principle, not lead to the humiliation of the particles.

Energo-phase (Energo-medium, Power-medium) is global medium which executes all force acts on the particles and masses in real World. In the basic (undisturbed) state, the Energo-phase is an isotropic high-density gas-like (possibly 
also quasi-fluid) medium formed by its own fundamental particles referred to as the energions which are spinless and massless. These particles are very small, they move in all directions at speeds close to the speed of light and can only be of two types: the Left and Right what linked with the appropriate direction of their own rotation. General state of the energions within Energo-medium is determined as doubly degenerate. Super-high mobility and not-inertial behavior of the energions allow the particles and masses to move relatively freely in Energomedium when this medium is in basic (undisturbed) state.

According to the Physical Triad Concept all forces direct action on the particles and masses, which are implemented in the Energo-phase are the forces of the so-called "Dark Energy" which represent the zones of non-equilibrium States in Energo-medium in the form of oblasts of local pressures of the energions. The formation of "Dark Energy" in Energo-medium is induced by spinor fields, i.e. fields of charged particles. In so doing all variety of spinor fields including gravitational fields don't have any real of power significance. They only play the role of intermediaries exerting influence on state of the Energo-medium and inducing formation of "Dark Energy" in it. Namely "Dark Energy" is real forces factor, performing the whole dynamics of particles and bodies, as in the scale of the Universe (the movement of galaxies, stars, planets and other objects) so and in the microcosm, for example, the dynamics of the spinor particles within a Physical masses of such as in atoms, nucleons, etc.

The spinor fields which are generate by spinor particles, depending on nature of particles are divided on fields of Matter and Antimatter. Fields of Matter represent himself flows of the energions which is polarized on their linear and angular pulses. Antimatter fields are antipoles to of fields of Matter and are manifested by processes of depolarization of those energions flows that material particles emit in the form of fields of Matter. In addition, the particles as of Matter, so and Antimatter can be both electric and magnetic. Depending on the nature and sign of the source charges the plus and minus signs can be assigned to the corresponding spinor fields.

The fields emitted by charges of different signs and nature create in the region between charges the zones of negative or positive "Dark energy". It is the sign and the magnitude of the forces "DE" that determines the status of the interaction, i.e. attraction between particles or their repulsion, as well as the force level of interaction, for example, electric, magnetic or weak. As will be shown in the article, the last interaction is realized within the framework of electromagnetic dipoles and is an example of a classical electromagnetic interaction.

Note 3. The Energo-medium can also be called as the Energo-ether, and instead of the ethereal particles known as Amers in composition of the Physical Triad were been injected particles of the Energions. The introduction of the prefix "Energo" in the composition of the name third component of PT and its particles is a reflection of the global force significance of this phase in the dynamics of particles and bodies in the real World. The author's articles show that "Dark 
Energy" which is the driving force of the Universe, is a force manifestation of the Energo-medium.

In detail the concept of the Physical Triad of the real World and the questions connected with the fields of Matter and Antimatter, as well as the essence of the "Dark energy" contain in two editions of the author's book [10], and reviewed in [11].

\section{Nucleons, Positron and Neutrino Are of the Electromagnetic Atomic-Shaped Structures and Are Sources the Gravitational Field}

The nucleons, positrons and neutrinos, like atoms, are varieties of physical mass the characteristic feature of which is the ability to radiate the gravitational field and, as a rule, to gravitate toward the Earth. Processes of the attraction or gravitation realized are realized between masses (bodies) which emitting a para-gravitational field. In our case, this is, for example, the Earth and the nucleon (see Section 2). The atomic-shaped device of all types of Mass and, in particular, of nucleons, positrons and neutrinos is of the natural and only possible organization of electric and magnetic spinor particles, what can radiate the gravitational field. The gravitational fields of nucleons are formed, mainly, by their electromagnetic envelope. At the same time, the gravitational contribution of their nuclei should not be ignored. Consequently, all of Masses, which are structured, exclusively, in the atomic-shaped forms, can be defined as gravitational-forming structures.

Thus, the nucleons can be defined as the "small atoms", and positrons and neutrino as "very small atoms" with its own shells and nuclei. Under this their shells are electromagnetic i.e. forming, mainly, by the nucleonic, positronic and neutrinos of the S-Gravitons which we will denote, respectively, as nS-Gravitons or nS-G, PS-Gravitons or PS-G, and $\mathcal{L S}$-Graviton or $\mathcal{L S}-\mathrm{G}$, where letters $\mathrm{n}$ and $\mathrm{P}$ is borrowed from words nucleon and Positron, and $v$ from the designation of neutrinos. The electric and magnetic particles in shells of nucleons, positrons and neutrinos which by tradition should be called quarks have charges of smaller magnitude than the charges of particles in atomic shells.

Note 4. The use of the name "quark" for the particles composing the electromagnetic shells of nucleons, positrons and neutrinos, require additional explanations. It is known that quarks are spinor particles (Standard Model), which have an electric charge multiple of e/3. They are part of hadrons and are not observed in the state free. The shells of atoms, nucleons, positrons and neutrinos, according to the concept developed by the author are electromagnetic, i.e. contain in their composition both electrical and magnetic fundamental particles. In addition, the charges of particles in the shells of nucleons, according to our results are multiples of $\mathrm{e}(\mathrm{g}) / 2$. Of course, such particles are not generally accepted theoretical quarks. Therefore it was necessary: either to introduce new names for particles in the shells of nucleons and others, or to use for them the name "quarks", but modernizing their parameters in relation to the new of physical 
circumstances. The last decision, according to the author, is the most rational. Since the "new quarks" in the structures of the shells of nucleons and in the shells of the positron and neutrinos differ in the values of the charges, of their designation, with respect to the corresponding masses, will be, respectively, $q_{m}$, $q_{e}$ and $q_{m}, q_{e}$. It is important to add that as the most probable candidates in the compositions of nuclei of nucleons, positrons and neutrinos, the author proposes only well-known particles that are unrelated ideologically to theoretical and new quarks.

The processes of formation gravitational field defined by vortex vector $\operatorname{rot}[\boldsymbol{E}-\boldsymbol{H}]$ and of vortex magnetic field defined as $\operatorname{rot} \boldsymbol{H}$, in the structures of the nucleons, positrons and neutrino are analogous to the processes of formation of these fields in atoms (see Figure 1). For example, so-called the magnetic moment of the neutron and possible magnetic moment of neutrino is form by the quarks magnetic dipoles which are not built into the in compounds of S-Gravitons, and like to the atomic conditions revolves on one of external orbitals their shells.

In determining the parameters and quantities of particles in nucleon structures, it is very important to introduce into our analysis such states gravitational field as the ferrogravitation and paragravitation (see Section 2 of this article). Depending from states of the gravitational field is being implemented wellknown processes of mutual attraction or repulsion of Masses. If an even number S-Gravitons is contained in the shell of an atom or nucleon, then the field that they emit is, as is a rule, is paragravitational, and the atom or nucleon is attracted to the Earth. An odd number of S-Gravitons in the shell of the atom or nucleon lead to the emission of a ferrogravitational field.

The author in his studies the found that the masses (bodies) emitting the ferrogravitational field repel from masses emitting a paragravitational field, for example, repel from the Earth. The shell of a hydrogen atom, which consists of one S-Graviton, is the source of the ferrogravitational field, and hydrogen is not attracted to the Earth, but is repelled from its. As known, hydrogen is volatile atoms, and of its volatility is determined by the effect of Gravitational levitation, i.e. pushing out Masses emitting the ferrogravitational field from the Earth's gravitational field [5] [6]. Based on the fact that the nucleons is attracted to the Earth, and also taking into account the magnitude of the charge of the nucleus of the nucleon, one can judge the amount of nS-G in the nucleon shell and, consequently, on number of quarks and magnitude their charges.

\section{Components Shell and Nucleus of Proton}

As stated above, the nucleons are "small atoms", i.e. consist of an electromagnetic shell and a nucleus. Therefore, in determining the structural features of nucleons, as well as the parameters of particles that can enter into their compositions it is necessary to be guided by the conditions that were developed by the author as applied to the electromagnetic device of atoms [5] [16].

1) The shells of nucleons, like the shells of atoms, are electromagnetic. They 
consist of electric and magnetic spinor particles, which should be called quarks. These particles are massless, since individually they do not represent a mass and, therefore, can't form their own gravitational field. The sources of the gravitational field in the shells of nucleons are the nucleonic S-gravitons, which consist of magnetic and electric (quark) dipoles and are denoted as nS-gravitons or nS-G.

2) The charges of the quarks that make up the shells of proton and neutron must be equal in absolute magnitude and, in accordance with their dipole state, should have plus and minus signs. It can also be assumed that the magnitude of the charge of quarks in the shells of nucleons should be substantially smaller than the charges of electron or magneton.

3) The so-called anomalous "magnetic moment" of neutron, like the "magnetic moment" of atoms, is formed by dipoles composed of magnetic quarks that are not embedded in $\mathrm{nS}-\mathrm{G}$ structures. These dipoles, like the magnetic dipoles of so-called magnetic atoms, populate the outer orbitales of the neutron shell, which, by analogy with magnetic atoms, can be designated as $d$ or $d_{n}$.

4) Nucleons are attracted to the Earth, i.e. they are sources of the paragravitational field (PGF). The main source of PGF is the shells of nucleons, which implies presence two of $\mathrm{nS}-\mathrm{G}$ on one its orbitals. In this case, the direction of the vortex vectors $\operatorname{rot}[\boldsymbol{E}-\boldsymbol{H}]$ of these $n S-G$ must correspond to their mutual compensation. By analogy with atomic shells, the orbitals of the nucleonic shells which are filled compensated nS-Gravitons can be designated as $s$ or $s_{n}$.

5) The author's researches are allowed the made the assumption that in the compositions of the nucleon nuclei can, as a rule, be realized the varieties of Physical Mass. At the same time, the nuclei of nucleons, like atomic nuclei, should have positive electric charge, and also to emit a gravitational field and, hence, to participate in the gravitational interaction. In addition, again by analogy with atoms, other particles that are not charged with an electric charge can exist in nucleus compositions. The last condition, as will be below, concerns nucleus of neutron.

Taking into account the conditions noted above, it is possible to compile probable representations about the parameters of specific particles in the structures of shells and nuclei of nucleons. First of all, the nucleon shells must be electromagnetic, i.e. consist of electric and magnetic quarks. The latter assertion contradicts the existing theory (Standard Model) according to which the valence quarks that determine the composition of nucleons can have only electric charges of $1 / 3 \mathrm{e}$ and $2 / 3 \mathrm{e}$, where $\mathrm{e}$ is the electron charge. In addition, according to the existing theory virtual (sea) quarks-antiquarks and gluons enter into the composition of nucleons.

Consider on the example a proton two options for the possible composition of its shell:

1) The magnitude of the electric and magnetic charges of the quarks in the proton structure is meets of Standard Model, 
2) Quarks have electric and magnetic charges the magnitudes of which differs from those accepted in existing theories.

Variant 1. In the proton shell are contained, so-called, the lower or d-quark with charge $-1 / 3$ e and the corresponding it antiquark with charge $+1 / 3$ e. In addition, in the composition each of the nucleonic nS-G, in addition to the pair of marked electric quarks, there must necessarily be a pair of magnetic quarks with charges $-1 / 3 \mathrm{~g}$ and $+1 / 3 \mathrm{~g}$, where $\mathrm{g}$ is the charge of the magneton, i.e. of the magnetic spinor in the atomic shell. We recall that the magnetic and electric charges of particles in atomic shells correspond to the condition $\mathrm{e}=\mathrm{g}$. By analogy with the atomic electromagnetic device the sum of the negative electric charge of the quarks in the nucleon shell should be equal to the positive charge of the proton nucleus, i.e. known proton charge $\mathrm{e}^{+}$. According to the last condition, the proton shell should include three of nS-G. However, as shown above, an odd number of $\mathrm{nS}-\mathrm{G}$ in atomic shells is accompanied by the formation of a ferrogravitational field. The latter field as is shown by the author's experimental studies is accompanied not by attraction but by repulsion from Earth. Thus, in the shells of nucleons there must be an even number $n S-G$, because only in this case the nucleon is able to form a paragravitational field and ensure the classical gravitational interaction (the so-called attraction) of the nucleon to Earth. Conclusion: quarks with electric charges of $1 / 3 \mathrm{e}$, not to mention $2 / 3 \mathrm{e}$ charges, can't be considered as the constituents of nucleon shells.

Variant 2. On one orbital in the proton shell there is a pair nS-G, and the electric and magnetic charges of the quarks, in this case, can be $1 / 2 \mathrm{e}(\mathrm{g}), 1 / 4 \mathrm{e}(\mathrm{g})$, $1 / 8 \mathrm{e}(\mathrm{g})$ and so forth. The last condition implies the realization of mutual compensation of the vortex vectors $\operatorname{rot}[\boldsymbol{E}-\boldsymbol{H}]$ of nS-Gravitons in pairs which corresponds to the compensation of electron spins in atomic shells. Only in this case the gravitational field emitted by such a shell, with a high degree of probability, can be a paragravitational field, and the proton will be attracted to the Earth. It is easy to see that the most probable is the last variant according to which the proton shell includes an even number of nS-Gravitons on one orbital and the maximum charge of quarks in the proton shell will be $1 / 2 \mathrm{e}(\mathrm{g})$. The question of the real number of $n S-G$ in the composition of nucleon shells requires additional studies, since there is no clarity in the true values of the quark charges in the nucleon shells, i.e. $1 / 2 \mathrm{e}(\mathrm{g})$ or $1 / 4 \mathrm{e}(\mathrm{g})$...? In further analysis of the nucleon structures we take as the basis the charges of quarks equal to $1 / 2 \mathrm{e}(\mathrm{g})$.

The most likely candidate for the role of the proton nucleus, in the author's opinion, should to consider a well-known positron whose charge is $\mathrm{e}^{+}$. As noted above in this article, the positron is not a true antielectron. The positron has an electrical charge equal $\mathrm{e}^{+}$, but that's all that can to connect it to the true antielectron. In all other aspects these are very far apart particles. The positron participates in the classical gravitational interaction, i.e. is a species of Mass. As for the antielectron it is the massless spinor particle that can't create its own electromagnetic vortex (gravitational) field. The positrons should be classified as stable 
particles. They are most often observed in the decay of radioactive nuclei, as well as in the irradiation of substance with gamma quanta. As already noted, the participation of the positron in the classical gravitational interaction allows us to assume that this particle has atomic-shaped electromagnetic structure. The shell of the positron can be made up of electric and magnetic quarks, and the magnitude of their charges must being less than the charges of proton quarks. It is possible that this is $1 / 4 \mathrm{e}(\mathrm{g})$ or $1 / 8 \mathrm{e}(\mathrm{g})$. As the nucleus of a positron one should consider such a spinor particle as a true antielectron with charge $\mathrm{e}^{+}$.

The positron and antielectron, as the author's studies have shown, are different particles and it necessary determining their notations. The symbol $\mathrm{e}^{+}$by which usually has denoted the positron must be attributed to the true antielectron. The possible symbol for the designation of a positron can be $\mathrm{P}^{+}$

\section{Structure of the Neutron and Nature of Weak Interaction}

The structure of the neutron was first experimentally investigated by R. Hofstadter by scattering of high-energy electrons on neutrons entering into deuterons [17]. According to the results of these studies, the neutron consists of a heavy nucleus and its surrounding shell, the so-called "meson fur coat". The representation of the Hofstadter neutron structure correlates perfectly with the definition of a neutron as a "small atom", which is used by the present author. Considering that the neutron mass is close to the proton mass it can be assumed that the electromagnetic part of the neutron shell creating the gravitational field is identical to this component in the proton structure. Thus, two nS-Gravitons in the neutron shell occupy one s-orbital, and their vortex vectors $\operatorname{rot}[\boldsymbol{E}-\boldsymbol{H}]$ are in a state of mutual compensation. As already noted, the charge of quarks in the shells of nucleons was assumed to be $1 / 2 \mathrm{e}(\mathrm{g})$.

However, there are significant differences between the proton and neutron shells. First of all, the neutron exhibits the so-called anomalous magnetic moment which is realized in the processes it magnetic scattering. There is no similar moment in the proton. The author's research has shown that the magnetic moments universally accepted in theory are theoretical prostheses behind which the real magnetic charges are hidden [5]. So-called magnetic moments in atomic shells form magnetic dipoles which are not part of atomic S-Gravitons and fill external d-orbitals. Quite similar is the situation and with the magnetic moment of the neutron. Consequently, the definition of a neutron as "small atom" should be extended to its definition as of the "small magnetic atom".

It should be noted that as far back as 1936 the question of the possibility of the existence of magnetic dipoles in the structure of a neutron was raised by F. Bloch [18]. According to his assumption it is these magnetic dipoles are responsible for the magnetic moment of neutron. Unfortunately, in the future, Bloch did not become to develop this direction and moved to the camp of supporters of the electric nature of magnetism and magnetic moment.

The magnetic quark dipoles (are indicated in the article as $\mathrm{md}_{\mathrm{q}}$ ) which form 
so-called the anomalous magnetic moment of neutron, as well as the use of the positron as the proton nucleus make it possible to make an assumption about the possible composition the nucleus of neutron. In the author's opinion in the nucleus of neutron, besides a positron, it is necessary participation a particle with a negative but magnetic charge $\mathrm{g}^{-}$, i.e. of the magneton which also can be called the "magnetic electron". This particle is not antagonistic to the positron and, at the same time, it is capable of providing the necessary interaction between the magnetic dipoles in the neutron shell and the nucleus. In addition, an important condition that must be found within such an association is the substantial compensation of the electric charge of a neutron, i.e. charge of positron and, as a consequence, the manifestation of a weak interaction between the magneton and positron. These, the last conditions as will be shown below, are connected with the circumstances of the neutron decay.

A structural device of the proton and neutron are shown in Figure 2. Two nS-G (large circles), that inhabit on one s-orbital in the shells of nucleons represent the electromagnetic density of nucleons which is responsible for their gravitational manifestations. The arrows nearby with $\mathrm{nS}-\mathrm{G}$ determine the directions of their vortex vectors $\operatorname{rot}[\boldsymbol{E}-\boldsymbol{H}]$ which in pair are mutually compensated. Small circles on the d-orbital in the neutron shell correspond to quark magnetic dipoles $\left(\mathrm{md}_{\mathrm{q}}\right)$. The arrows nearby with the dipoles show the direction of the vortex magnetic fields produced by them determining by the vortex vectors $\operatorname{rot} \boldsymbol{H}$. Unlike from vectors $\operatorname{rot}[\boldsymbol{E}-\boldsymbol{H}]$, the vortex vectors $\operatorname{rot} \boldsymbol{H}$ in the neutron shell are uncompensated. It is this vortex magnetic field created by the magnetic dipoles was substituted in the existing theory on so-called magnetic moment of the neutron. As noted above, the proton nucleus is the positron (denoted as $\left.\mathrm{P}^{+}\right)$. The nucleus of the neutron, in the author's opinion, is the pair: the positron $\left(\mathrm{P}^{+}\right)$and the magneton $\left(\mathrm{g}^{-}\right)$.

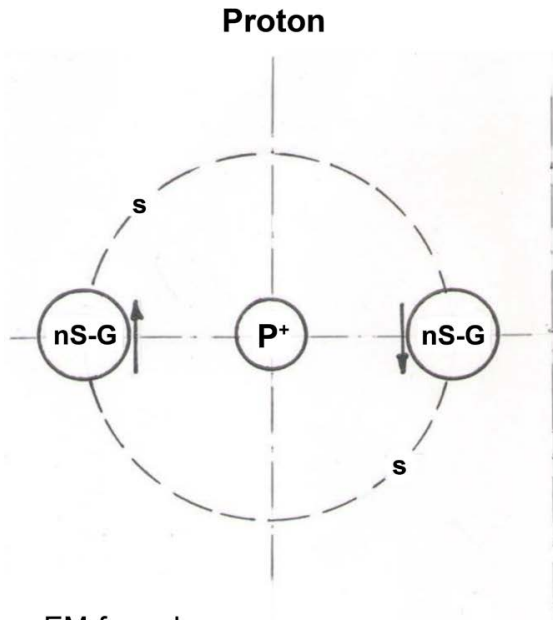

EM-formulas:

$\mathbf{S}^{2}(\mathbf{n S}-\mathbf{G})$

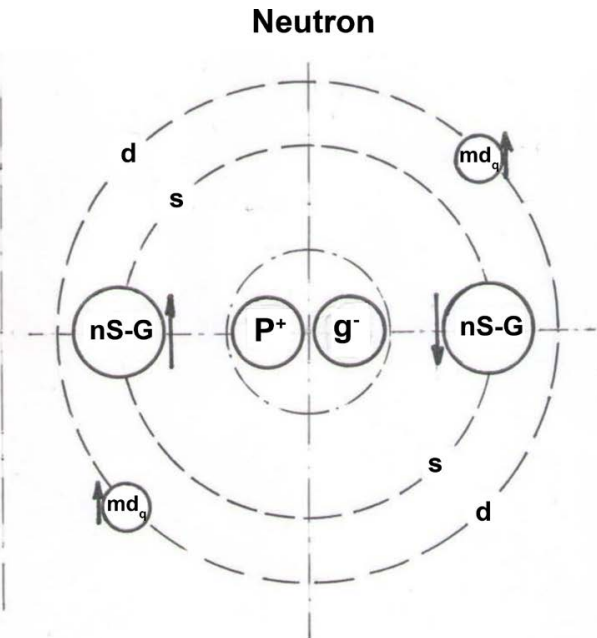

$\mathrm{S}^{2}(\mathrm{nS}-\mathbf{G}) \mathrm{d}^{2}\left(\mathrm{md}_{\mathrm{q}}\right)$

Figure 2. The schemes structural device of the proton and neutron as well as is the electromagnetic formulas (EM-formulas) their shells. 
The pair: positron and magneton which, in the author's opinion, constitute the nucleus of neutron is the weak electromagnetic dipole in which a weak interaction is realized. It is the weak coupling (weak interaction) between the particles in the composition of this dipole leads, ultimately, to the decay of the neutron. The situation with fields and interactions of the electric charges in a pair $\mathrm{e}^{+}$ $\div \mathrm{e}^{-}$is well known. Similarly, this situation is realized and in pair of magnetic charges $\mathrm{g}^{+} \div \mathrm{g}^{-}$. However, how the spinor fields manifest themselves in of electromagnetic pairs of charges, for example, in a pair of charges $\mathrm{e}^{+} \div \mathrm{g}^{-}$is a big mystery. The problematics of this issue is not difficult to understand, if we take into account that the real magnetic charges (magnetic analogs of the electric charges) in existing theoretical concepts (Standard Model) are categorically absent. Within the framework of the present article we consider possible schemes for the distribution strength of fields and interactions in a pairs of charges $\mathrm{e}^{+} \div$ $\mathrm{g}^{-}$and $\mathrm{e}^{+} \div \mathrm{g}^{+}$which must be referred to as the electromagnetic (weak) dipoles.

In his books and articles (see [10] [11]), and also briefly in Section 4 of this article, the author showed that the force effects within all possible bispinors and dipoles are determined by the action of the forces of "Dark Energy" ("DE” ) which is realized in the region between the charges under the influence of their fields. This "Dark energy" can be both negative and positive. The negative "Dark energy" formed in the region between the charges is responsible for the so-called attraction of charges and the positive "DE" for their repulsion. According to the researches of the author, the fields emitted by the charges of Matter ( $\mathrm{e}^{-}$and $\mathrm{g}^{-}$) and charges of Antimatter $\left(\mathrm{e}^{+}\right.$and $\left.\mathrm{g}^{+}\right)$, correlate with each other as opposite fields or anti-fields. In accordance with the sign of the charge, it is possible to assign the corresponding sign to the field emitted by this charge. Thus, the vectors of electric fields strength emitted by the electric charges $\mathrm{e}^{+}$and $\mathrm{e}^{-}$can be designated as $\boldsymbol{E}^{+}$and $\boldsymbol{E}^{-}$, and the vectors of the magnetostatic field strength emitted by the magnetic charges $\mathrm{g}^{+}$and $\mathrm{g}^{-}$as $\boldsymbol{H}^{+}$and $\boldsymbol{H}^{-}$. It is important to note that the pair of charges the electromagnetic dipole $\left(\mathrm{e}^{+} \div \mathrm{g}^{-}\right)$represents the charges of the neutron nucleus and, as will be shown below, also the neutrino nuclei.

The Nature and signs of charges and fields, as well as the sign and the force status of the "Dark Energy" which form in the compositions of the dipoles under consideration, are shown in Figure 3.

Features in the distribution strength of fields in electromagnetic pairs (Figure 3(b) and Figure 3(b)) are determined by the fact that the fields in these pairs, in the author's opinion, are the weakly commuting fields (WCF). Namely, the weak impact of the WCF of the charges composing the electromagnetic dipoles on the state of the Energo-medium leads to the formation of a weak "Dark energy" and weak interaction. For example, the weak impact of the fields $\boldsymbol{E}^{+} / \boldsymbol{H}^{-}$on the state of the Energo-medium in the region between the charges in the pair $\mathrm{e}^{+} \div \mathrm{g}^{-}$ is manifested by "weak" electric charge of the neutron observed in the experiments [19] [20] (are given below in the article). 


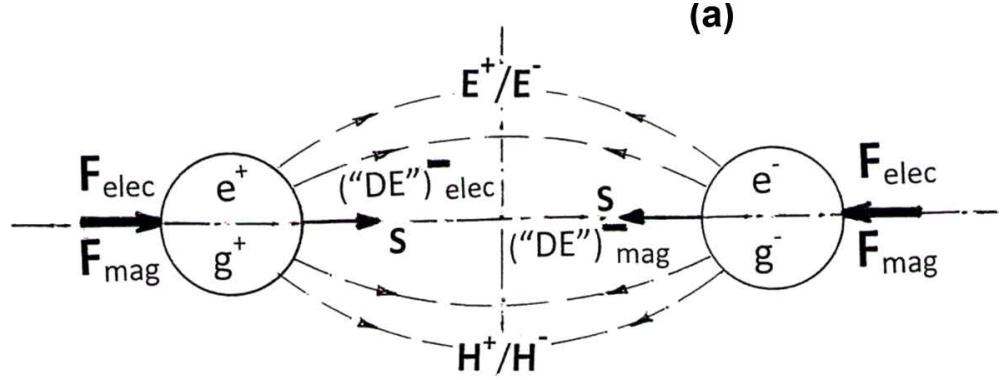

(b)

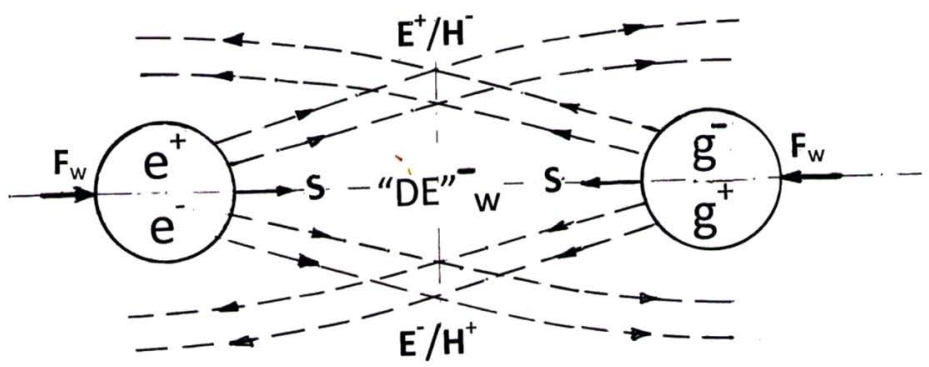

(c)

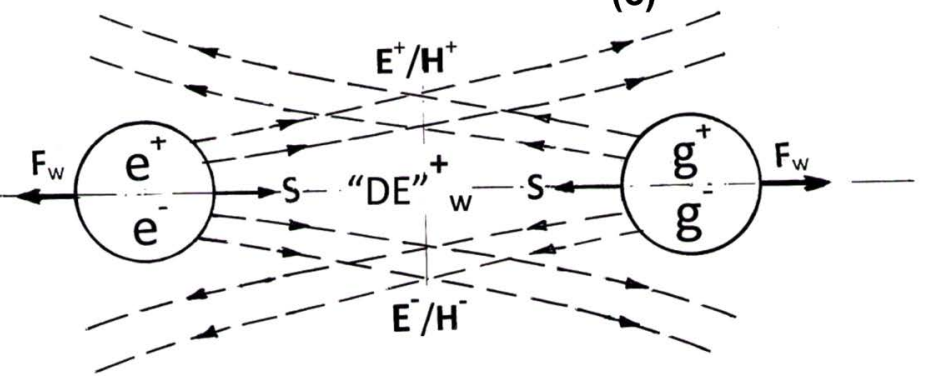

Figure 3. The schemes states of the fields and forces in dipoles which are consist of homogeneous spinor particles (electric and magnetic) (a) as well as in electromagnetic (weak) dipoles: $\mathrm{e}^{+} \div \mathrm{g}^{-}$and $\mathrm{e}^{-} \div \mathrm{g}^{+}$(b), $\mathrm{e}^{+} \div \mathrm{g}^{+}$and $\mathrm{e}^{-} \div \mathrm{g}^{-}$(c). The spinor fields, forces, as well as the signs and the force status of the "Dark Energy" are indicated in Figure 3. The symbols "w" for the force vectors and "DE" notations, is taken from the term "weak". By means of $\mathbf{S}$ the vectors spins of particles in the dipoles are indicated.

Graphically, the effect of WCF of electromagnetic dipoles on the state of the Energo-medium leading to weak interaction is shown in Figure $3(\mathrm{~b})$ as a weak curvature of the field lines to the center of the Figure (weak attraction), and in Figure $3(c)$ as a weak curvature of the lines from the center of the Figure (weak repulsion).

The concept of a "weak charge" in the dipole composition must be associated with the corresponding force effects that are realized when the dipole field is impact on of the Energo-medium. For example, an electric charge of $\mathrm{e}^{+}$and its field $\boldsymbol{E}^{+}$in the compositions of the homogeneous and heterogeneous (electromagnetic) dipoles are induce different in magnitude force manifestations, which is determined by the efficiency impact of fields in the composition of dipoles on the Energo-medium. If electron in the electric dipole shows to a force effect corresponding to a known charge e, then in the composition of the elec- 
tromagnetic dipole, the force manifestation of the electron will correspond to the so-called of weak electron charge according to the condition $e_{e f} \ll e$, where $e_{e f}$ is the effective charge of the electron. Effective charges of particles in the compositions of dipoles of different nature can differ by many orders of magnitude. The different efficiency of charges presented above in the compositions of dipoles of different nature is, in the author's opinion, a new effect, since magnetic charges, i.e. the true sources of the magnetostatic field determined by the magnetic polar vector $\boldsymbol{H}$ are stubbornly ignored in of the physical science for more than a hundred years.

Note 5. It is note that charges in the nucleus of neutron, due to the weak connection between them, can constitute the so-called short dipole whose state can be described as $L \approx d$, where $L$ is the distance between the charges, and $d$ is the size of the charged particle. We recall that the state of the classic bispinor, i.e. of the ground state of the spinor particles is characterized by a dense pressing in a pair of charges of opposite signs that are homogeneous in nature. As repeatedly stress in the article, the particles in this state are tightly pressed against each other. They do not manifest themselves by spinor fields and are very difficult to detect.

Thus, a weak interaction is realized exclusively in electromagnetic pairs of the spinor particles, i.e. in electromagnetic dipoles. As for the so-called electromagnetic interactions, generally accepted in the theory, they are realized exclusively in dipoles consisting of homogeneous charges. These latter interactions are either electrical or magnetic but not electromagnetic.

In the framework of electromagnetic dipoles is being implemented mutual suppression of the effectiveness of the impact the fields of charges on the state of the Energo-medium which leads to a minimization of the magnitude, of "DE" which is formed in the region between charges. This mutual compensation of the efficiency of charges of electromagnetic dipoles determines the force action between particles, which is called weak interaction.

It is necessary to clearly understand that the manifestation a charge of particles is perceived solely through the influence of their fields on Energo-phase and the implementation of the corresponding "DE" in it. As for the fields realized in the framework of weak (electromagnetic) dipoles they initiate the formation in the region between the charges of the so-called of weak "DE", which and explains the fact of the weak charge of neutron, observed experimentally. The chief circumstance which forces us to use the term "weak" when considering the physics of electromagnetic dipoles, as already noted above, are connected with so-called the weak commutation of electric and magnetic fields in their compositions.

This last assumption is confirmed by experiments in which a weak electric charge is detected free neutron, which according to various estimates is as: $Q_{n}=(-1.9 \pm 3.7) \times 10^{-18}\left|Q_{e}\right| \quad[19]$ to $Q_{n}=(-1.5 \pm 2.2) \times 10^{-20}\left|Q_{e}\right|$ [20]. The electric charge of a neutron, observed in experiments can be called a weak charge. 
An important result of the author's researches is the conclusion that weak interaction is realized in the compositions of electromagnetic dipoles and is exclusively type of electromagnetic interaction. These interactions are is determined by set of fields realized between charges of the electromagnetic dipoles and, as a consequence, the values and signs of the forces "DE" formed under their action. As for the spinor fields that initiate the "DE", they are the electric and magnetic fields of particles of Matter and Antimatter. Earlier the author mistakenly assumed that weak interaction is realized by means of the so-called fast spinor fields whose velocities in space exceed the speed of light [10] [11]. Such a conclusion was made under the influence of the well-known theory of electroweak interaction, according to which in the hot Universe electromagnetic and weak interactions are combined into a single electroweak interaction.

\section{The Decay of Neutrons, the Interconversion of Nucleons in Atomic Nuclei, and a Strong Interaction}

The comparison the structures of the proton and neutron described above, discover that their common element is the electromagnetic structural unit in the form of a proton. The shell of the neutron differs from the proton shell in that, in addition to the pair nS-G in there are there are magnetic charges in the form of quark magnetic dipoles, which are responsible for the so-called anomalous magnetic moment of the neutron. The difference in the nuclei of the proton and neutron is that in the latter, apart from the positron, there is a magneton or the "magnetic electron". In the decay of neutron is being implemented a "purification" of the structure of proton, from "ballast" in the form of the quark magnetic dipoles in shell, and also from the magneton in nucleus.

The neutron decay which should be referred to as $\beta_{\mathrm{m}}$-decay can be written in the form: $\mathrm{n}^{\circ} \rightarrow \mathrm{p}+\mathrm{g}^{-}+2\left(\mathrm{q}_{\mathrm{m}}^{-} \div \mathrm{q}_{\mathrm{m}}^{+}\right)+\gamma$. Designations products of the neutron decay: $\mathrm{p}$-proton, $\mathrm{g}^{-}$-magneton or "magnetic electron", $\mathrm{q}_{\mathrm{m}}^{-}$and $\mathrm{q}_{\mathrm{m}}^{+}$are magnetic quark and antiquark and as well as in some cases, the $\gamma$-quantum. The coefficient 2 in front of the quark magnetic dipole $\left(\mathrm{q}_{\mathrm{m}}^{-} \div \mathrm{q}_{\mathrm{m}}^{+}\right)$is determined by the charges of the quarks, which are taken as $1 / 2 \mathrm{~g}$, where $\mathrm{g}$ is the charge of the magneton $(\mathrm{g}=\mathrm{e})$. The designation of the supposed neutron decay as $\beta_{\mathrm{m}}$-decay is related to the fact that instead of an "electric electron", one should expect the output of a magneton, i.e. "magnetic electron" ( $\mathrm{m}=$ magnetic). The yield of a $\gamma$-quantum can serve as evidence of the perturbation of the electromagnetic shell when magnetic quarks leave the neutron composition upon its decay. The fact that not all neutron decays are accompanied by the release of a $\gamma$-quantum can mean that there are some, in this case anomalous, features of decays even in states of free neutrons.

Above in the article was cited data on the weak electric charge of a neutron. It was assumed that a weakening of charge efficiency $\mathrm{e}^{+}$of the positron in the nucleus of the neutron occurs as a result of the influence on its field of the field "magnetic electron". If this is possible, then we should expect a similar action of 
positron on the field of magneton and, taking into account of the symmetry of these effects, a weak of the magnetic charge of neutron also possible. In addition, the magnetic fields of the above-mentioned quark dipoles, which are responsible for the so-called the magnetic moment of the neutron can, to some extent, effect on a field of magneton. However, due to the vortex nature of such fields the last effect cant' be considered determinative.

Let us consider, in general terms, the conditions for recording the products of the decay of a free neutron. Registration of a protons and gamma quantum does not present any serious problem. The conditions for recording the "magnetic electron", i.e. magneton, in principle, do not differ from the registration of the "electric electron". The replacing an electron by magneton, in the plan of energy, is also not a problem, since here there is only a change in Nature of charge, i.e. replacing the left on right. The main problem is the registration of the magnetic quarks, which determines the anomalous magnetic moment of neutron. In his publications [1] [5], the author showed that the ground state of the spinor particles is the state of the bispinor, i.e. of the connected pair of spinor particles with charges of opposite signs. In this state, the spinor particles are tightly pressed together which leads to compensation of the activity of their spins. In this case, bispinors can't manifest themselves through spinor fields and are extremely difficult to detect.

It is important to supplement the above with the fact that these of quark magnetic dipoles perform a very important practical function in the neutron composition, answering because so-called the anomalous magnetic moment of the neutron. As for neutrinos, they are involved in existing theories as an energy "additive", which is designed to preserve the energy balance in the decay of a neutron. In addition, the neutrino is a very convenient object in terms of the popular explanation of why apart from protons and electrons and, occasionally, to the $\gamma$-quants, in practice, nothing is more observed in experiments with the decay of neutrons.

The existence of quark magnetic dipoles in shell of the neutrons and magnetic spinor particle (magneton) in its nucleus determines ultimately the neutron instability. In a free state, the neutrons decay after $\sim 15$ minutes after exiting the nucleus. However, as a part of atomic nuclei, a neutron is considered to be a stable particle. Proceeding from the physics of neutron and a proton of developed by the author, such a concept as the stability of a neutron in atomic nuclei seems highly questionable.

As shown above, to extract the proton from structure of neutron it is necessary to remove magnetic (quark) dipoles from its shell, and remove the "magnetic electron" from its core. On the other hand, in order to reconstruct a neutron, these dipoles and magneton must be introduced into the proton structure. In the author's opinion, it is precisely the complex of magnetic spinor particles $\left[2\left(\mathrm{q}_{\mathrm{m}}^{-} \div \mathrm{q}_{\mathrm{m}}^{+}\right)+\mathrm{g}^{-}\right]$which converts a neutron into a proton and, conversely, a proton into a neutron, creates the false impression regarding of stability the 
neutrons in atomic nuclei. In the decay of neutrons in the composition of atomic nuclei, the noted complex of the magnetic particles, leaving neutron, penetrates into one of the neighboring protons, converting it into a neutron. Thus, a constant number of protons and neutrons are maintained in atomic nuclei and is created the illusion the stability the neutrons in atomic nuclei. The process described above for the conversion of a proton into a neutron is written in the form: $\mathrm{p}+\left[\mathrm{g}^{-}+2\left(\mathrm{q}_{\mathrm{m}}^{-} \div \mathrm{q}_{\mathrm{m}}^{+}\right)\right] \rightarrow \mathrm{n}^{\circ}$.

It is important to recall that still in 1935 the Japanese physicist H. Yukawa predicted that between the proton and neutron in the nucleus is possible active exchange by the particles which called $\pi$-mesons or pions [21]. The forces that are realized as a result of the exchange by mesons, in Yukawa's opinion, are the nuclear forces (NF) that bind nucleons in atomic nuclei.

Mesons, according to the existing theory, consist of an equal number of quarks and antiquarks, what, somehow, harmonize theirs with the complex of magnetic particles $\left[\mathrm{g}^{-}+2\left(\mathrm{q}_{\mathrm{m}}^{-} \div \mathrm{q}_{\mathrm{m}}^{+}\right)\right]$, which the author presented as possible exchange complex between neutron and proton in nuclei. Of course, unlike the mesons adopted in the existing theories, the last "mesons" are composed of magnetic particles and can be referred to as magnetic mesons or m-mesons, and also as $\mathrm{m}_{\mathrm{m}}$.

It can be assumed that in the exchange of magnetic mesons between nucleons, the exchange energy is realized, which, in this case, should be referred to as internucleon exchange energy. Namely this energy, according to Yukawa's theory, should be responsible for the strong interaction. The processes of mutual transformations of nucleons in nuclei, with the help of magnetic mesons, can be denoted as $\mathrm{n}^{0} \leftarrow \mathrm{m}_{\mathrm{m}} \rightarrow \mathrm{p}$.

However, not everything is so simple in the real physics of strong interaction. The author's discovery of magnetic spinor particles (magnetic charges) in the structures of atoms and substance, as well as the subsequent discovery by him of the electromagnetic (vortex) nature of the gravitational field, made significant corrections to his representations of the strong interaction physics. Since 2001, the author in his publications [1] [4] [5] [6] argued that the nucleons are "small atoms" with its own shell and nucleus. Nucleons emit their own (nucleon) gravitational field, which is the paragravitational field and is manifested as in the processes of attraction between nucleons so and, for example, in attractions of nucleons to the Earth.

The gravitational fields of neighboring nucleons induce zones of negative "Dark energy" in the region between them with. At short distances between nucleons in nuclei, the compression forces created by such a "Dark energy" can be quite significant. In addition in the processes compression of nucleons in atomic nuclei, an essential contribution is made by the EM-shell of atoms, creating at their center, i.e. on nuclei, the gravitational pole contributing to the formation in the core region of the additional contribution of negative "Dark energy". If the force effects caused by nucleons gravitational fields are tend to 
extend to neighboring nucleons then the forces of the "Dark Energy" associated with the gravitational pole affect all nucleons in the nucleus.

The fact that the atomic shell participates in the processes of nucleon compression in nuclei is detected when the shell is removed from the nucleus, which, as a rule, leads to destruction of nuclei. Of course, one should not forget about the electrostatic repulsion of protons from each other. The described complex of spinor fields (electric, magnetic, gravitational) acting on the "Energo-medium" induces in the core region the "Dark Energy", which in sum is negative. This negative "DE" and is the force factor, which is responsible for the stability of the atomic nuclei. As for the exchange energy, so for the author does not have any ideas as to how this energy is consistent with the state of the Energo-medium and, therefore, with "Dark energy".

Note 6. In the author's opinion, the concept of the exchange energy as the content of nuclear forces is used, exclusively, because of the absence of more significant, and importantly, more natural of mechanisms in the theoretical arsenals. It is possible that the introduction of the gravitational factor, the general physics of which developed by the author and is very accessible in the representations, can leave such confusing notion as the exchange energy "without the main work". It is also important to note that gravitational interactions between masses at ultra-small distances $(<1 \AA)$ have not been studied, and these forces can be very significant.

Summing up the considerations regarding the participation of gravitational fields and gravitational "Dark energy" in the composition of nuclear forces, the author does not exclude the participation in their composition and "meson" factor. However, as will be shown below, its possible contribution to the strong interaction depends on the mass numbers of the elements and can be significant only in the case of large atomic Masses.

In process of mutual transformations of nucleons in nuclei should not expect the yield of $\gamma$-quanta, since they are rarely observed even in the decays of free neutrons. The question of the mean lifetime of neutron in nuclei is discussed below in Chapter 9. Here we can say that this parameter is in nuclei is much smaller than that of the lifetime of a free neutron and essentially depends on the mass numbers of the atoms.

In Physics of the neutron decay, discussed above with the participation of real magnetic particles there is a very important question: what, in the end, pushing the neutron to decay? In the author's opinion, the decays of neutrons initiate neutrino, which are considered in all theoretical analyzes of these processes. At the same time, it should be recognized that neutrino in this process is external agent that initiates decay, but is not a real product of neutron decay. The Impact of a neutrino on a neutron and its compulsion to decay is realized both in the free state of the neutron and in the composition of the nuclei. The neutron decays as result of the interconversion between a positron and a neutrino in a neutron nuclei is considered below in Chapter 9 . 


\section{The Atomic-Shaped Structures of Positron $\left(\mathrm{P}^{+}\right)$ and Neutrino ( $v$ ) and Their Interconversion in the Neutron Nuclei}

The process of neutron decay, as well as the interconversion between a neutron and a proton in atomic nuclei, was discussed above in Chapter 8. In order to clarify the mechanism of neutron decay it is necessary to determine, first of all, the structural device of the positron and the neutrino. It is precisely these Masses (particles), in the opinion of the author, which determine an actions, leading to the decay neutron is realized. If the positron is part of the neutron nucleus, then, according to the author, the neutrino is external agent which interacting with a positron in composition of the neutron nucleus and compelling the neutron to decay. This interaction is realized in the form of an interconversion between a positron and a neutrino which, in some way, resembles the above described process of interconversion between a proton and a neutron in atomic nuclei. It is the interconversion between the positron and the neutrino that creates the conditions for the neutron decay, both in atomic nuclei and in state of free neutron.

The positron and neutrinos are the varieties of mass and participate in gravitational interaction. In Section 5 of this article it was shown that only the masses that always have an atomic-shaped electromagnetic device can form a gravitational field. Consequently, the positron and the neutrino must have an electromagnetic shell and a nucleus.

The shells of these particles are formed by the corresponding S-Gravitons, composed of electric and magnetic quarks, whose charges are smaller than the charges of quarks in the shells of nucleons. The possible values of the charges of quarks in the shells of positron and neutrino can be $1 / 4 \mathrm{e}(\mathrm{g})$ or $1 / 8 \mathrm{e}(\mathrm{g})$. An obligatory condition in the realization of the interconversion between a positron and a neutrino is the equality of the magnitude of the charges of quarks in the shells of these particles.

As the nucleus of the positron, we should consider an electric particle with a charge of $\mathrm{e}^{+}$, i.e. the true antielectron. This is a fully possible option, since namely the real antielectron according to the author's version, forms an electric bispinor with an electron, under the conditions of their so-called annihilation. As for the neutrino nucleus, in its composition, in addition to the antielectron it is necessary to include a magneton, i.e. the magnetic particle with charge $\mathrm{g}^{-}$. It is important to note that the participation of the magneton in the neutrino core assumes the existence of uncompensated magnetic quark dipoles in the neutrino shell. By analogy with the neutron, in this case, one should expect the realization of the so-called the anomalous magnetic moment of the neutrino.

The complex of magnetic particles participating in the processes of the interconversion of the positron and the neutrino is written in the form: $\left[\mathrm{g}^{-}+4\left(\mathrm{q}_{\mathrm{m}}^{-} \div \mathrm{q}_{\mathrm{m}}^{+}\right)\right]$. Unlike the complex of particles that participate in nucleon transformation processes, in the latter case, magnetic quarks (marked in red) are involved in the neutrino shell with charges that are of multiple of less than the 
charges of quarks in nucleon shells. The transformation processes between neutrino and positron can be written in form: $v+\left[\mathrm{g}^{-}+4\left(\mathrm{q}_{\mathrm{m}}^{-} \div \mathrm{q}_{\mathrm{m}}^{+}\right)\right] \rightarrow \mathrm{P}^{+}$and $\mathrm{P}^{+}+\left[\mathrm{g}^{-}+4\left(\mathrm{q}_{\mathrm{m}}^{-} \div \mathrm{q}_{\mathrm{m}}^{+}\right)\right] \rightarrow v$. The coefficient 4 in front of the magnetic quarks dipole $\left(\mathrm{q}_{\mathrm{m}}^{-} \div \mathrm{q}_{\mathrm{m}}^{+}\right)$corresponds to the condition that the charge of the quarks in the shells of the positron and the neutrino is $1 / 4 \mathrm{e}(\mathrm{g})$.

The possible structural schemes and electromagnetic formulas of positron and neutrino show on Figure 4. The large circles in Figure 4 are the designate SGravitons ( $v S-G)$ corresponding to these masses, which, in pairs, are located on each of two s-orbitals the positron and neutrino shells. The orientations of the vortex vectors $\operatorname{rot}[\boldsymbol{E}-\boldsymbol{H}]$ of $v S-G$ inhabiting each s-orbital (1s and 2 s) are marked by arrows and meet the conditions for their mutual compensation. Since the values of the charges of the quarks in the structures positrons and neutrinos are equal, for S-Gravitons in composition their shells identical designation $v S-G$ is adopted. By small circles in Figure 4 are denoted the quarks magnetic dipoles $\left(\mathrm{md}_{\mathrm{q}}\right)$, which in the shell of neutrino are responsible for the so-called anomalous magnetic moment and are populate the d-orbital. Note that in Figure 4 (Neutrino) subscripts q в составе обозначения $\left(\mathrm{md}_{\mathrm{q}}\right)$, for technical reasons, are shown in black.

The names of the sublevels s and $d$ are set by analogy with atomic configurations, according to which the particles on the orbitals of the s-levels meet the conditions for compensation of particle spins, and the d-orbitals include spinor particles, which may be uncompensated. The pair: $\mathrm{e}^{+}$and $\mathrm{g}^{-}$which, in the author's opinion, constitute the nucleus of neutrino is of weak electromagnetic dipole. The weak coupling is between particles in composition of this dipole provide the possibility of interconversion neutrino with a positron.

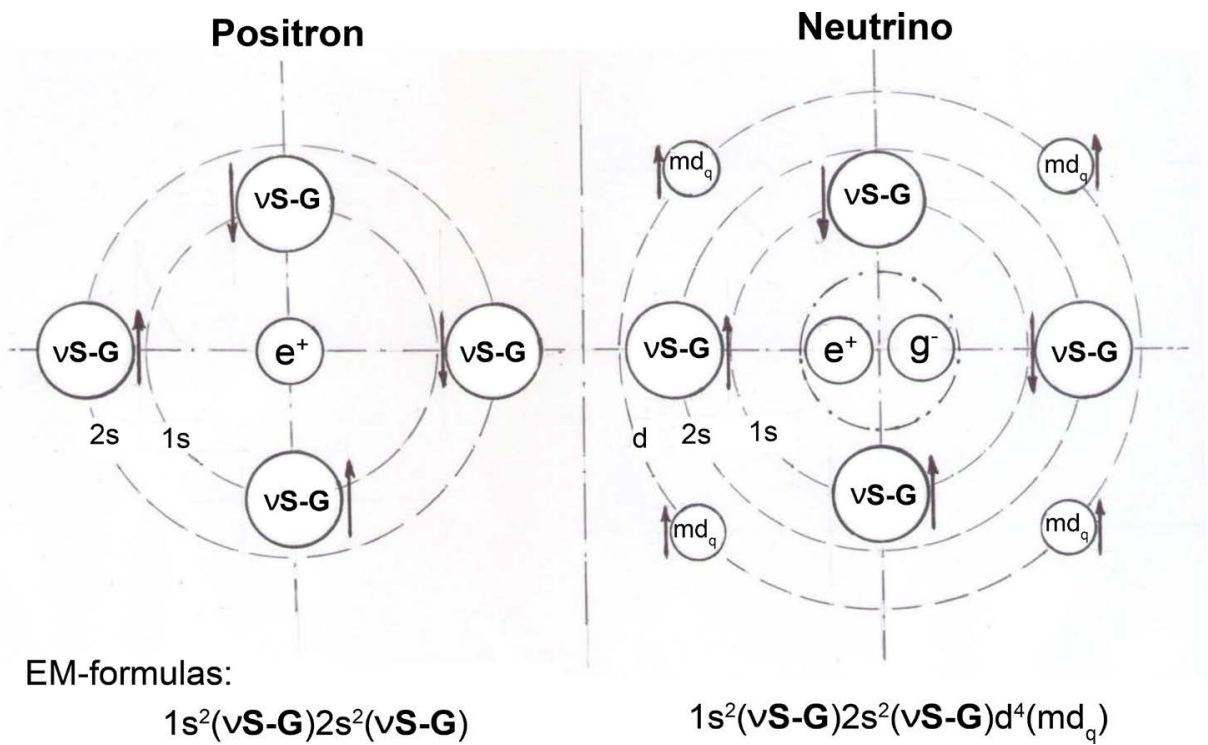

Figure 4. The structural schemes and EM-formulas of the positron and neutrino. The arrows in Figure 4 placed next to the magnetic dipoles $\left(\mathrm{md}_{\mathrm{q}}\right)$ show the direction of the vortex vectors $\operatorname{rot} \boldsymbol{H}$ of the dipole fields. The circuits shown in Figure 4 correspond to the values of the quark charges accepted by equal to $1 / 4 \mathrm{e}(\mathrm{g})$. 
Neutrino, after realization of interconversion with a positron, is recreated as a Phoenix bird and can, in principle, conduct decays of neighboring neutrons in atomic nuclei. An interesting question: does a certain neutrino "work" in the atomic nucleus on an "on-going basis", repeatedly initiating the decay of a neutron or is it the "work of hands" of various neutrinos?

The resolution of the latter question will allows us to draw certain conclusions relative to the possible frequency the interconversions between nucleons in atomic nuclei. If the decay of free neutrons determined by the density of the corresponding neutrinos in a given region of space, then in a multiple impact on neutrons of one neutrino in atomic nuclei period of decay neutrons should be significantly smaller, than the period of the decay of free neutrons. In this case, everything depends on the number of neutron decays which fall to the share of one neutrino "constantly working" in atomic nuclei. The relationship between period of decay of a free neutron $(t)$ and the period of their decay in atomic nuclei $(T)$, can be written, in the most general form, as: $T=t / K$, where $K$ is the average number of neutron decays that performs a one neutrino in a particular nucleus. It is not difficult to see that the value of $K$, which can be related to the effective neutrino scattering cross-section on neutrons in an atomic nucleus, will differ substantially for atoms with different mass numbers.

Estimating, in the most general form, the situation with the decay of neutrons in atomic nuclei, it should be concluded that the period of neutron decay in nuclei will decrease with the growth of their mass numbers. In addition, it is important to note that the energies of primary and secondary neutrinos that "work" with neutrons in atomic nuclei is equal, which will allow us to consider more thoroughly the energy of the processes of neutron decays.

The results of the author's research, which are the contents of this article, allow us to draw the following conclusions concerning the situation associated with the states and interactions of a neutron and a neutrino.

1) The neutron and neutrino are so-called the weak (friable) particles, what is determined by weak interactions between electric and magnetic charges in their nuclei. It is these weak interactions that determine the decays of these particles, which are described in detail in this article.

2) The neutrino oscillations detected in known studies [22] [23] is a serious argument in the favor of the fact that the neutrino is a variety of Mass and, if we follow the author's conception, has an atomic-shaped structure. Neutrino this is the so-called weak or friable Mass, the state of which can change under the influence of external factors, for example under the influence of electromagnetic fields. By weak elements in structure of neutrinos are both interactions between the charges of particles in its nucleus, and the state of its magnetic moment. It is precisely changes in the states of the marked elements that can be manifested in the observed neutrino oscillations.

3) The atomic-shaped structure of a neutrino assumes that this particle, in its natural essence, corresponds, rather, to the Majorana's type of particles [24] but 
not Dirac's.

It must be added that there is no basis for asserting that the interconversion of neutrino and positron takes place only in the nucleus of a neutron. This process can, in principle, be realized and in the proton core but due to the high stability of the proton it does can't lead to it the decay.

\section{Conclusions}

The interactions in atomic nuclei are subject of research of numerous theoretical schools and directions, which, however, rely solely on an electrical basis and do not wish, even at the level of physical idea, to consider the existence of real magnetic charges. As a result of this vicious restriction it is impossible to determine either the real physics of the gravitational field or the device of its sources. Introduction of real magnetic charges as well as of true antielectrons in the fundamental physical representations radically change the theoretical concepts that were developed without taking into account existence these particles in the structures of atoms and substance. The author's research has shown that the real magnetic charges are, that is, approximately, half of all of the fundamental particles of the real World. Therefore, correction of the existing physical concepts with the introduction of real magnetic charges in the theoretical analysis should be very significant.

Of course, the "absence" of magnetic charges and true antielectrons in physical representations forced the theorists to invent instead of them various theoretical prostheses. For example, the true antielectrons were replaced by Dirac holes and positrons, and the magnetic dipoles in atomic shells by magnetic moments. However these prostheses could not provide a full value replacement for these real particles that led to an extraordinary complication of modern theoretical physics. In his numerous publications, the author showed that the acknowledgment of real magnetic spinor particles (magnetic charges) and true antielectrons not only liberates physical science from numerous false and even mystical constructions, but also allows us to describe many complex effects and manifestations on language of the classical physics.

The electromagnetic structures of the atoms, nucleons, positrons and neutrino, real physics and mechanism of the formation of gravitational field, interconversion of proton and neutron in nuclei, the true antielectrons in the shells of atoms and much more, all this is the logical output from the author's experimental studies in which real magnetic poles and real antielectrons were discovered in the atoms and substance.

In addition, the legalization of magnetic charges and true antielectrons allows to discover new effects and new directions, which can't be detected when these particles are ignored. An example of this is the author's discovery of the effect of Gravitational levitation [12] [13] [14] [15], as well as of the effect Magnetoelectric induction, presented by the author in [25].

The atoms, nucleons and positrons, in the author's opinion, represent all that 
we call the Physical Masses in composition of substance. At each transition within the framework this System of atomic-shaped elements, for example, from atom to proton, are implement a significant transformations of shells and nuclei, with the simplification of their structure, but with the mandatory preservation of their atomic-shaped form. It is possible that the positron is the final "point" of possible varieties of Masses, forming a substance, when moving along a line of descending dimension. Of course, there is still a neutrino, which is also of Mass. However, this particle is most likely not a component of the substance. At the same time, neutrino is of paramount element of the Physical "merry-go-round" with interconversion of the Masses in the Universe.

Note 7. Base provisions of general physics with real magnetic charges, developed by the author, were presented at the international PIERS 2009 Moscow Conference "Progress in Electromagnetics Research" [26] [27].

\section{Acknowledgements}

The author expresses deep gratitude to Elena Sizova and Vitaly Sizov which are for many years helped and supported author what, in many respects, contributed of the his research activities.

The author is Grateful to technical specialists: A.A. Davydov, N.V. Koshelev, A.I. Lomakin, E.V. Moiseenkov and S.E. Staritsin, which provided technical support for the main part of experiments (period 1969-1986), connected with his research of real magnetic charges in atoms and substance.

\section{References}

[1] Sizov, R.A. (2001) New Presentation of Nature Magnetism, Gravitation and Nuclear Forces of Bonding. Akademizdat-Center "Science", Moscow.

[2] Sizov, R.A. (1970) Solid State Physics, 13, 2081-2088.

[3] Sizov, R.A. (1971) Journal of Experimental and Theoretical Physics, 60, 1363-1370.

[4] Sizov, R.A. (2008) Electric and Magnetic Spinor Particles Are Structure-Forming Components of Mass and Electromagnetic Source Gravitation. Akademizdat-Center "Science", Moscow.

[5] Sizov, R.A. (2015) Journal of Modern Physics, 6, 1013-1022. https://doi.org/10.4236/jmp.2015.68106

[6] Sizov, R.A. (2017) Journal of Modern Physics, 8, 1072-1086. https://doi.org/10.4236/jmp.2017.87069

[7] Sizov, R.A. (2015) Journal of Modern Physics, 6, 2280-2289. https://doi.org/10.4236/jmp.2015.615232

[8] Maxwell, J.C. (1873) Treatise on Electricity and Magnetism. Volume 1-2, Clarendon Press, Oxford.

[9] Ehrenhaft, F. (1942) Journal of the Franklin Institute, 233, 235-255. https://doi.org/10.1016/S0016-0032(42)90311-9

[10] Sizov, R.A. (2011/2012) Matter, Antimatter and Energo-Medium-Physical Triad of the Real World. Akademizdat-Center "Science", Moscow.

[11] Sizov, R.A. (2016) Journal of Modern Physics, 7, 558-572. https://doi.org/10.4236/jmp.2016.76059 
[12] Sizov, R.A. (2015) Journal of Modern Physics, 6, 1591-1601. https://doi.org/10.4236/jmp.2015.611161

[13] Sizov, R.A. (2012) Levitation as a Spread Force Manifestation of Natural Ferrogravitation. Akademizdat-Center "Science", Moscow.

[14] Sizov, R.A. (2013) Transference of People and Loads in the Terrestrial Space by Means of Technical Ferrogravitation. Akademizdat-Center "Science", Moscow.

[15] Sizov, R.A. (2013) Generation and Technical Application of Ferrogravitation (The Collection of the Invention of the Author in Area the Gravitophysical and Gravitochemical Technologies). Akademizdat-Center "Science", Moscow.

[16] Sizov, R.A. (2016) Journal of Modern Physics, 7, 2374-2397. https://doi.org/10.4236/jmp.2016.716206

[17] Hofsadter, R. (1957) Annual Review of Nuclear Science, 7, 231-316. https://doi.org/10.1146/annurev.ns.07.120157.001311

[18] Bloch, F. (1937) Physical Review, 51, 994.

[19] Shull, C.G., Billman, K.W. and Wedgwood, F.A. (1967) Physical Review, 153, 1415-1422. https://doi.org/10.1103/PhysRev.153.1415

[20] Gähler, R., Kalus, J. and Mampe, W. (1982) Physical Review D, 25, 2887-2894. https://doi.org/10.1103/PhysRevD.25.2887

[21] Yukawa, H. (1935) Proceedings of the Physico-Mathematical Society of Japan, 17, 48-57.

[22] McDonald, A.B., Klein, J.R. and Wark, D.L. (2003) Scientific American, 288, 40-49.

[23] Kearn, E., Kajita, T. and Totsuka, T. (1999) Scientific American, 281, 65-71.

[24] Majorana, E. (1937) Il Nuovo Cimento, 5, 171-184. https://doi.org/10.1007/BF02961314

[25] Sizov, R.A. (2017) Journal of Modern Physics, 8, 2374-2397. https://doi.org/10.4236/jmp.2017.86061

[26] Sizov, R.A. (2009) Electric and Magnetic Spinor Particles are the Electromagnetic Source of Gravitation. Theory and Experiments, The Report at PIERS 2009 Moscow.

[27] Sizov, R.A. (2009) Magnetic Particles (Magnetons) Are Structural Components of Atoms and Substance, Immediate Sources of Magnetic Field. Theory and Experiments, The Report at PIERS 2009 Moscow. 